Documentation et bibliothèques

DOCUMENTATION BIBLIOTHEQUES

\title{
Pour une vision globale du développement des services de bibliothèques et de documentation Synthèse des écrits récents et considérations générales
}

\section{Réal Bosa}

Volume 22, numéro 3, septembre 1976

URI : https://id.erudit.org/iderudit/1055314ar

DOI : https://doi.org/10.7202/1055314ar

Aller au sommaire du numéro

\section{Éditeur(s)}

Association pour l'avancement des sciences et des techniques de la documentation (ASTED)

ISSN

0315-2340 (imprimé)

2291-8949 (numérique)

Découvrir la revue

Citer cet article

Bosa, R. (1976). Pour une vision globale du développement des services de bibliothèques et de documentation : synthèse des écrits récents et considérations générales. Documentation et bibliothèques, 22(3), 123-129. https://doi.org/10.7202/1055314ar

\section{Résumé de l'article}

Après avoir souligné le cloisonnement historique des diverses catégories de bibliothèques au Québec et souligné le développement récent d'une remise en cause de cet état de choses visant à une plus grande planification provinciale, l'auteur relie, par le biais d'un certain nombre de sources pertinentes, ce phénomène à des mouvements similaires apparus un peu partout à travers le monde - souvent sous l'impulsion de l'Unesco - et surtout aux États-Unis. Il s'attache en particulier, pour ce dernier pays, au rapport de la National Commission on Libraries and Information Science publié en 1975. De ces divers documents, l'auteur tire quelques principes généraux essentiels à la mise en oeuvre d'une véritable planification nationale et fait ressortir, dans le rapport de la Commission, la philosophie de base ainsi que les principales recommandations. Ces mouvements constituent une interrogation posée à la bibliothéconomie québécoise.
Tous droits réservés (c) Association pour l'avancement des sciences et des techniques de la documentation (ASTED), 1976
Ce document est protégé par la loi sur le droit d'auteur. L'utilisation des services d'Érudit (y compris la reproduction) est assujettie à sa politique d'utilisation que vous pouvez consulter en ligne.

https://apropos.erudit.org/fr/usagers/politique-dutilisation/ 


\title{
Pour une vision globale du développement des services de bibliothèques et de documentation
}

\author{
Synthèse des écrits récents et considérations générales
}

\author{
Réal Bosa \\ École de bibliothéconomie \\ Université de Montréal
}

\begin{abstract}
Après avoir souligné le cloisonnement historique des diverses catégories de bibliothèques au Québec et souligné le développement récent d'une remise en cause de cet état de choses visant à une plus grande planification provinciale, l'auteur relie, par le biais d'un certain nombre de sources pertinentes, ce phénomène à des mouvements similaires apparus un peu partout à travers le monde - souvent sous l'impulsion de l'Unesco - et surtout aux États-Unis. II s'attache en particulier. pour ce dernier pays, au rapport de la National Commission on Libraries and Information Science publié en 1975. De ces divers documents, l'auteur tire quelques principes généraux essentiels à la mise en œuvre d'une véritable planification nationale et fait ressortir, dans le rapport de la Commission, la philosophie de base ainsi que les principales recommandations. Ces mouvements constituent une interrogation posée à la bibliothéconomie québécoise.
\end{abstract}

After having underlined (1) the historical separateness of different types of libraries in Québec and (2) the recent questionning of this state of things in view of province wide planning, the author traces this same phenomenon through the pertinent literature in other countries where similar movements occurred often under the investigation of Unesco. He pays particular attention to the philosophy and main recommendations of the Report of the National Commission on Libraries and Information Science in the U.S., published in 1975. From these documents the author draws some general principles that are essential to true national planning. These movements and principles constitute a challenge to Québec librarianship.

Después de haber subrayado la división histórica de las diferentes categorías de bibliotecas en Québec, y subrayado el desarrollo reciente de una vuelta a este estado para llegar a una más grande planificación provincial, el autor relaciona este fenómeno a movimientos similares aparecidos por todo el mundo - muchas veces por impulsión del Unesco - y sobre todo en los Estados Unidos. Por este último país, se concentra en particular al informe de la National Commission on Libraries and Information Science (1975). De estos diferentes documentos, el autor coge algunos principios generales esenciales a la verdadera planificación nacional, y hace resaltar, en el informe de la Comisión, la filosofia de base y las recomendaciones principales. Estos movimientos constituyen una interrogación preguntada a la biblioteconomía quebequense.

Considéré dans son ensemble, le développement des bibliothèques relevant de l'État du Québec s'est effectué jusqu'à maintenant selon des voies parallèles et sur la base de principes directeurs émanant des services gouvernementaux respectifs.

Ces cheminements autonomes, voulus comme tels au point de départ, visaient à bien délimiter les rôles spécifiques de deux grandes catégories de bibliothèques: les bibliothèques publiques et les bibliothèques d'enseignement, et à contrecarrer les fausses économies de certains employeurs. Quant à l'approche directive, elle s'explique par bon nombre de raisons tenant autant à l'état de 
la profession qu'à des situations de fait à l'époque de la prise en main par l'État du secteur de l'éducation.

Cette façon de concevoir les choses a conduit à la constitution d'ensembles cloisonnés (bibliothèques publiques d'une part, bibliothèques d'enseignement d'autre part) et à une sclérose de la communication et de l'organisation inter-bibliothèques tant au niveau régional que local. Le constat établi par les spécialistes Aubry-Denis concernant les bibliothèques publiques de la région de Montréal est éloquent à cet égard: "On peut dire que d'une manière générale il n'existe pas de relations formelles ou contractuelles entre les bibliothèques de la région de Montréal." ${ }^{1}$

Il y a bien eu, dira-t-on, des efforts louables entrepris au niveau de la Fédération des collèges classiques (puis de la Commission des directeurs de bibliothèques de CEGEP), de la CREPUQ ${ }^{2}$, des bibliothèques gouvernementales et de la Bibliothèque nationale du Québec en vue d'amorcer des dialogues, d'établir des concertations. Les initiatives entreprises, dont certaines fort valables, ont cependant rarement mis en question les structures établies.

Or, comme nous le verrons plus loin, bien des pays, dont les États-Unis qui nous ont servi de modèle au plan de l'organisation bibliothéconomique, remettent actuellement en cause les structures établies ou tout au moins se préparent à les modifier considérablement compte tenu de la conjoncture professionnelle et économique récente.

\section{Des interrogations collectives}

II serait certes instructif de retracer l'évolution de pensée de nos associations professionnelles concernant une approche globale du développement des bibliothèques.

Certains thèmes des congrès annuels de I'ACBLF et de l'ASTED ont permis, au cours de la dernière décennie, d'explorer des sujets pertinents à notre propos: "L'ACBLF et la coopération internationale" (1967), "Le livre centre de la documentation accessible à tous" (1972), "Des bibliothèques pour le milieu» (1973) et celui du prochain congrès qui s'insère dans la ligne des précédents tout en marquant une note plus agressive.

L'un de ces congrès qui portait sur le thème spécifique de notre exposé nous inté-

\footnotetext{
1. Texte tiré du rapport - à paraître sous peu- sur les bibliothèques publiques de la région de Montréal.

2. Sous-comité des bibliothèques, Conférence des recteurs et des principaux des universités du Québec.
}

resse particulièrement: "La planification du développement des bibliothèques" (1966). $Y$ furent présentés en particulier des textes fort évocateurs; le premier, celui de la conférence d'ouverture présenté par Paule Rolland-Thomas, traite surtout de coopération entre bibliothèques et évoque l'attitude qui prévalait à l'époque, et qui règne encore, à l'égard du rôle de l'État:

"Si nous acceptons la planification dans le sens défini par Robert, nous invoquons fort probablement un genre de dirigisme, voire d'étatisme dans toutes nos tâches professionnelles...” ${ }^{3}$

"La planification, c'est-à-dire la mainmise gouvernementale sur toutes nos activités professionnelles est-elle vraiment la solution? N'atteignerions-nous pas les mêmes objectifs par la coopération? $n^{4}$

Le deuxième texte, celui du président de l'ACBLF, Patrick Allen, établit la nécessité d'une planification d'ensemble pour le Québec:

"Sans un plan ordonné et concerté de développement, sans une coordination consentie des efforts, la croissance normale des bibliothèques sera compromise. Le coût d'aménagement et d'opération sera prohibitif et le financement impossible. Nous aboutirons à un chaos dont plusieurs générations souffriront.... ${ }^{5}$

"J'invite... tous les bibliothécaires à réfléchir sur la nécessité d'un plan concerté et librement consenti, non seulement entre bibliothèques d'une même catégorie (bibliothèques de régionales, de collèges et d'universités, etc.) mais entre bibliothèques de catégories différentes. $m^{6}$.

L'auteur est explicite: "ll faut donc planifier, il faut de la planification à l'échelle provinciale.n II souligne l'importance de la mise sur pied d'un organisme de planification pour l'ensemble du Québec, sans toutefois se référer au rôle de l'État dans ce domaine.

3. "La coopération entre les bibliothèques", in ACBLF, La planification du développement des bibliothèques. Rapport des travaux du $22 e$ congrès tenu à Moncton du 14 au 17 août 1966, Montréal, 1967, 14.

4. Ibid., 19.

5. "La planification du développement des bibliothèques", Bulletin de l'ACBLF, vol. 12, no 1 (mars 1966), 3.

6. Ibid. 


\section{Un concept nouveau: la planification nationale}

S'il existe une théorie de la planification applicable à l'entreprise, et surtout l'entreprise commerciale, il est permis d'affirmer par contre qu'au niveau des bibliothèques la "planification... n'a pas reçu jusqu'ici toute l'attention qu'elle mérite et, si on la considère en fonction des besoins de la recherche et du développement, on constate qu'elle en est, à ce point de vue également, à ses débuts"?

Or il s'avère, compte tenu de la conjoncture économique mondiale et des développements au plan international en matière de bibliothéconomie et de documentation, que "les années 1970 seront probablement celles de la planification des services de bibliothèques" ${ }^{8}$.

Le développèment de systèmes mondiaux tels I'UNISIST, le CBU, le NATIS, et la décision de bon nombre de pays de souscrire et de participer à ces systèmes, appellent de soi la nécessité d'une planification nationale, cette dernière devant conduire par voie d'entraînement à la planification à titre individuel des bibliothèques et services de documentation.

\section{Ecrits en matière de planification nationale et internationale}

Un certain nombre de documents ont été publiés, en particulier à partir des années 1970, concernant de façon directe cette question. Citons des études plus théoriques: de Carlos Victor Penna, La planification des services de bibliothèques et de documentation (2e éd. rev. et augm. par Philip H. Sewell et $H$. Liebaers, Paris, Unesco, 1971, 178 p.); de J.H. d'Olier et B. Delmas, La planification des infrastructures nationales de documentation, de bibliothèques et d'archives; esquisses d'une politique générale (Paris, Unesco, 1974, 328 p.); les comptes rendus de réunions d'experts ${ }^{9}$ organisées par l'Unesco sur la planification nationale en Amérique latine (Quito, 1966), en Asie (Colombo, 1967), en Afrique (Kampala, 1970) et dans les pays arabes (Caire, 1974); les documents de travail

7. Carlos Victor Penna, La planification des services de bibliotheques et de documentation, $2 \mathrm{e}$ éd. rev. et augm., Paris, Unesco, 1971, p. 48.

8. Ibid., 49.

9. Voir le Bulletin de l'Unesco à l'intention des bibliothèques. et le compte rendu' ${ }^{10}$ de la Conférence intergouvernementale sur la planification des infrastructures nationales en matière de documentation, de bibliothèques et d'archives, organisée par l'Unesco et tenue en septembre 1974 à Paris; le compte rendu" du congrès annuel de 1974 de la FIAB portant sur le thème "National and International library planning»; le relevé des expériences récentes et en cours dans plusieurs pays paru dans Advances in Librarianship ${ }^{12}$, ainsi qu'un document d'importance majeure qui constitue en fait le programme établi au plan national américain par la National Commission on Libraries and Information Science (NCLIS), Toward a National Program for Library and Information Services: Goals for ACtion $^{13}$.

A ces études, il convient d'ajouter un certain nombre d'écrits périphériques, i.e. touchant des aspects qui entrent en considération dans l'exercice même de la planification nationale. Nous en énumérons ici quelquesuns parmi les plus récents:

Withers, F.N. Normes pour l'établissement des services de bibliothèque; enquête internationale. Paris, Unesco, 1974. $450 \mathrm{p}$. (Documentation, bibliothèques et archives. Études et recherches, 6).

Gardner, Frank M. Législation relative aux bibliothèques publiques: étude comparative. Paris, Unesco, 1971. 285 p. (Documentation, bibliothèques et archives. Études et recherches, 2).

Manpower in the Field of Documentation and Library Services. Paris, Unesco, 1973. $163 \mathrm{p}$.

Schütz, Harold (comp.). Le rôle et l'organisation d'un centre national de documentation dans un pays en voie de développement, par un groupe de travail FID/DC. Paris, Les Presses de I'Unesco, 1976. 230 p. (Documentation, bibliothèques et archives. Études et recherches, 7).

10. "Conférence intergouvernementale sur la planification des infrastructures nationales en matière de documentation, de bibliothèques et d'archives, Paris, 23-27 septembre 1974», Bulletin de l'Unesco à l'intention des bibliothèques, vol. 29 (janvier-février 1975), 2-16.

11. Ce document doit être publié sous peu.

12. F.E. Morhrhardt et C.V. Penna, "National planning for library and information services", in Advances in Librarianship, 5 (1975), 61-106.

13. U.S.A., National Commission on Libraries and Information Science, Washington, 1975, 106 p. 


\section{Justification de la planification nationale}

Les auteurs énumèrent un certain nombre de raisons justifiant une planification nationale: l'accroissement phénoménal de la masse documentaire, le rythme de développement de la recherche, la hausse des coûts d'organisation et d'exploitation des ressources documentaires, une prise de conscience dans bon nombre de pays en ce qui a trait à l'instrument essentiel au développement constitué par l'information scientifique et technique.

A ces raisons s'ajoute celle de l'impact de la technologie; la planification nationale est primordiale dans ce domaine, et ce, à plusieurs égards:

"D'abord la technologie est coûteuse et le gouvernement doit dès le début s'engager à long terme pour assurer la stabilité du programme. En second lieu, la technologie étant complexe, il est indispensable qu'il existe au niveau national une direction technique éclairée si l'on veut que toutes les institutions intéressées coordonnent efficacement leurs activités. En troisième lieu, la technologie est spécialisée et l'évolution d'un programme national d'information doit être synchronisée avec l'enseignement technique dispensé aux personnes qui en assureront l'application. Enfin, en raison du caractère novateur de la technologie, son introduction modifie invariablement les habitudes et exige que l'on se penche sur les problèmes du recyclage de l'utilisateur.»14

Les exigences mêmes de la planification internationale par l'établissement de systèmes mondiaux d'information commandent une planification d'ensemble au niveau de chaque pays.

II conviendrait enfin, pour être plus complet, d'ajouter une dernière raison, directement reliée à la situation des pays dits en voie de développement. Le grave problème des masses humaines croissantes qui n'ont pas encore accès au mot imprimé et la menace de retour à l'analphabétisme qui guette les personnes récemment alphabétisées si l'on néglige d'instaurer, dans le milieu, des supports dits culturels, commandent dans ces

14. Conférence intergouvernementale sur la planification des infrastructures nationales en matière de documentation, de bibliothèques et d'archives. Paris, 23-27 septembre 1974, Rapport final, Paris, Unesco, p. 31. pays des actions d'ensemble de la part des gouvernements concernés.

\section{Exigences de la planification nationale}

Une conception qui tend à se répandre assez largement et qui est clairement formulée comme énoncé de principe dans le document de la NCLIS veut que, dans un pays, les ressources documentaires relevant des organismes publics et privés soient considérées comme des biens collectifs nationaux («national resource») destinés à contribuer, à divers plans, à l'avancement de la nation. Et ces ressources doivent être organisées en vue de leur accessibilité à l'ensemble des citoyens.

Cette vision globale, ajoutée au fait qu'une proportion considérable de services de bibliothèques et de documentation relève de toute manière de l'Etat aux plans juridique et financier, commande que l'État assume un rôle majeur dans la planification nationale. Planification qui, selon C.V. Penna, ne peut se concevoir de façon isolée mais doit s'intégrer à celle des divers secteurs d'activités du pays: culturels, éducationnels, scientifiques, etc.

"C'est seulement à cette condition que les plans de développement des bibliothèques auront des chances d'être approuvés, en particulier dans les pays en voie de développement, qui doivent tirer le meilleur parti de leurs ressources. Faute de s'intégrer dans les plans de développement d'ensemble, il est peu probable en effet que ces projets bénéficient d'un appui national ou international ou d'une aide bilatérale et, même s'ils sont bien intégrés, il se peut que des pressions politiques et économiques contraignent à ne les réaliser que de façon fragmentaire. ${ }^{15}$

L'exercice de la planification implique des choix tant au niveau des objectifs que des moyens. La perspective étant globale, i.e. s'appliquant à l'ensemble des services de bibliothèques et de documentation, l'effort de planification nationale, surtout dans un pays développé, pourra se concentrer sur un certain nombre d'aspects essentiels et majeurs, quitte à évaluer dans une phase ultérieure le rendement de services existants et déjà structurés.

Il est évident, bien que peu d'auteurs insistent sur la question, que la garantie de réussite d'un plan national réside dans une

15. C.V. Penna, La planification..., 5. 
large consultation de la profession et des divers organismes concernés lors des diverses phases allant de l'élaboration jusqu'à l'évaluation des programmes mis en vigueur.

Un dernier aspect important, évoqué par Robert Vosper dans son exposé lors du congrès de 1974 de la FIAB, est qu'aujourd'hui plus que jamais la planification nationale doit se concevoir dans une perspective internationale:

"The national plan must strive to be compatible with other national programs and with developing international and global programs. In practice this means that national planning must go in the context of, and be constantly accompanied by, participation in international planning in order to achieve maximum interaction and communication.

Access to the knowledge and information resources of the entire world is essential to the development of every nation. This means that we must give entire support and encouragement to all efforts to provide international linkages and access." 16

\section{Engagements concrets}

Jetons un regard sur les actions récentes entreprises par l'Unesco et l'expérience américaine en cours en matière de planification nationale.

En septembre 1974 avait lieu, à Paris, une conférence intergouvernementale organisée par l'Unesco et portant sur la planification des infrastructures nationales en matière de documentation, de bibliothèques et d'archives. Cette conférence, qui regroupait 254 délégués de 86 États membres et 63 observateurs, était "chargée de formuler les concepts généraux qui se dégagent des travaux des conférences régionales sur la planification tenues en Amérique latine, en Asie, en Afrique et dans les États arabes et d'élaborer des directives générales en matière de politique et de méthodologie de la planification des services de documentation, de bibliothèques et d'archives". Les débats furent regroupés sous trois thèmes principaux: la planification intégrée des infrastructures, l'application de la technologie et la planification concernant le personnel.

Les participants, à la fin de la conférence, adoptèrent le concept général d'une planifi-

16. Texte diffusé lors du congrès de la FIAB en 1974 cation globale des infrastructures nationales, tel que formulé dans un document intitulé Systèmes nationaux d'information (NATIS): objectifs d'une action nationale et internationale. Les recommandations adoptées à cette conférence dégagent un certain nombre de principes et tracent, dans ses grandes lignes, le programme à suivre par les États en cette matière. II y est clairement défini, entre autres, ce qui suit:

- il revient à chaque État d'assumer la responsabilité de la planification nationale;

- la planification nationale doit se concevoir de façon globale et intégrée aux autres éléments du plan ou de la politique du pays;

- chaque Etat doit élaborer une "politique nationale d'information, reflétant les besoins de tous les secteurs de la communauté, et de l'ensemble de la communauté nationale»;

- "les fonctions de tous les services de documentation, de bibliothèques et d'archives doivent être coordonnées par l'intermédiaire d'un organisme central (ou de plusieurs) pour former le système national d'information (NATIS), afin que les ressources disponibles soient utilisées au mieux et que le système apporte la contribution la plus large possible au développement culturel, social et économique du pays; " 17

- «le plan national d'information doit prévoir les dispositions voulues par l'application de la technologie de l'information... dans l'intérêt de l'utilisation maximum des ressources existantes ainsi que de la compatibilité entre les systèmes et de la normalisation; 18

- «il faut agir sur le plan législatif le plus tôt possible afin d'appuyer la planification et la mise en place du système national d'information (NATIS). Cette législation doit porter sur la base conceptuelle du système et des éléments qui le composent, y compris tous les sous-systèmes spécialisés; ${ }^{19}$

17. Conférence intergouvernementale... Rapport final, 28-29.

18. Ibid., 30 .

19. Ibid., 31. 
- la planification nationale doit être conçue de façon à pouvoir intégrer la participation aux systèmes mondiaux qui se développent: tels I'UNISIST, le CBU.

L'adoption unanime de ce concept du NATIS a incité I'Unesco à inscrire à son budget pour l'année 1975-1976, à titre d'élément majeur de ses activités, les moyens de traduire ce concept en programme d'action de façon à promouvoir ce système et à aider les États membres (et en particulier les pays en voie de développement) à planifier et à développer leurs infrastructures nationales de manière à en assurer la coordination au niveau national et à préparer les bases d'une participation active dans les systèmes d'information mondiaux.

Les pays qui choisiront d'agir dans ce domaine possèdent désormais des lignes directrices et sont assurés de l'expertise professionnelle de I'Unesco. Le Canada, pays représenté à cette conférence par une délégation dont la composition a été ouvertement critiquée par I'ASTED, entend-il s'engager dans ce sens? Si oui, selon quelle approche, compte tenu de ses composantes politiques? La discrétion de la délégation canadienne, avant et après la conférence, ne nous permet pas de connaître les intentions canadiennes en cette matière.

Outre les actions entreprises par l'Unesco, il convient d'évoquer tout au moins brièvement l'initiative d'un pays fort développé en matière de ressources documentaires, de moyens et de structures: les États-Unis. Nous relèverons certains aspects particulièrement intéressants de l'expérience américaine, mais nous ne saurions trop recommander à nos collègues la lecture du document de la NCLIS mentionné plus haut.

La planification américaine a été rendue possible grâce à la mise sur pied d'une Commission largement autonome ( «an independent agency"), la National Commission on Libraries and Information Science, créée par une loi du Congrès et dotée de moyens financiers adéquats ${ }^{20}$.

Cette Commission, largement représentative dans sa composition, a choisi d'utiliser un ensemble de moyens pour remplir son mandat: commande d'études spécialisées, audiences auprès des divers milieux profes-

20. En 1974-1975 le budget de la Commission s'établissait à $\$ 500,000$ et le personnel à plein temps comptait neuf personnes, à l'exclusion des membres de la Commission. sionnels, tenue de colloques (ex. "Conference on user needs"), rencontres avec des représentants d'organismes fédéraux et des divers états, etc.

L'élaboration de la planification ne s'est pas effectuée en vase clos; un premier projet (draft proposal), élaboré en 1973 puis repris en 1974, fut largement diffusé dans les milieux gouvernementaux et professionnels.

Plusieurs aspects intéressants ressortent du rapport final déposé en 1975. La Commission établit clairement sa philosophie de base à savoir que toutes les ressources documentaires (des organismes publics ou privés) constituent un bien collectif («national resource»), et que tout citoyen américain doit avoir accès à ces ressources. L'objectif visé est centré nettement sur l'accessibilité des ressources documentaires à tout citoyen:

"To eventually provide every individual in the United States with equal opportunity of access to that part of the total information resource which will satisfy the individual's educational, working, cultural and leisure-time needs and interests, regardless of the individual's location, social or physical condition or level of intellectual achievement." 21

La Commission ne fait pas fi des structures et services existants: "local libraries are the first place in the Community where most people generally go to find information. Unless local systems, therefore, are strong and are supported by continuing, agressive, and dynamic leadership, they will be ineffective members in any program of nationwide scope." 22 L'un des objectifs envisagés est d'ailleurs le suivant: "to strenghten existing statewide resources and systems" ${ }^{23}$ et la structure nouvelle que propose la Commission, celle d'un réseau national de services (national net:vork) n'est pas perçue comme devant remplacer les réseaux existants: "the national network... would not be a monolithic and authoritarian superstructure, but would form a shelter and framework for families of geographic and functional networks developed and interconnected according to a comprehensive plan. ${ }^{24}$

21. National Commission on Libraries and Information Science, Toward a National Program for Library and Information Services: Goals for Action, Washington, 1975 , p. XI.

22. Ibid., 40 .

23. Ibid., 42

24. Ibid., XI. 
Notons enfin que la Commission accorde dans sa planification une importance assez grande aux services à des catégories particulières de la population; l'un des objectifs du programme proposé est spécifique à cet égard: "provide adequate special services to special constituencies, including the unserved. ${ }^{25}$

Le document émanant de la Commission est riche d'enseignements, tant au plan de l'approche philosophique que des attitudes à respecter dans l'élaboration d'une planification nationale.

\section{Une planification québécoise}

Les gouvernements qui choisiront désormais de repenser d'une façon globale le développement de leurs services de bibliothèques et de documentation pourront s'appuyer sur des bases théoriques plus solides (compte tenu des études mentionnées plus haut) et s'inspirer d'expériences valables faites dans un certain nombre de pays, dont les États-Unis, l'Angleterre et I'URSS, ce dernier pays ayant été le leader dans ce domaine.

L'engagement des pays ci-haut mentionnés, pays pourtant fort bien développés en matière de ressources et de services documentaires, vient réfuter l'argument à l'effet que la planification nationale s'impose uniquement dans les pays en voie de développement.

Dans un article publié récemment dans Argus $^{26}$ et portant sur les recommandations majeures de la Rencontre sur la bibliothéconomie québécoise, nous avons indiqué l'importance pour le gouvernement du Québec de s'engager dans cette voie de la planification de l'ensemble des services documentaires.

Nous connaissons les réticences de bon nombre de nos collègues face à une intervention accrue de l'État. Le comportement de ce dernier en regard de l'application de sa propre loi concernant notre Corporation professionnelle et des récentes négociations dans le secteur public laisse songeur. Par ailleurs, plus d'un bibliothécaire contestent le caractère de confidentialité rattaché aux corps consultatifs actuellement opérationnels au niveau gouvernemental et l'orientation

25. Ibid., 18.

26. Réal Bosa, "Rencontre sur la bibliothéconomie québécoise. Pour un développement planifié des bibliothèques et services de documentation", Argus, vol. 5, no 1 (janvier-février 1976), 3-4. énoncée par le Ministre des Affaires culturelles concernant la diffusion du livre par les bibliothèques publiques.

Nonobstant ces états de fait, les membres de la profession doivent s'interroger sur l'àpropos d'une planification d'ensemble pour le Québec et sur une conception de l'intervention de l'État, à la lumière des études publiées jusqu'à maintenant et des expériences entreprises à l'étranger.

Coopération, rationalisation et coordination: rêve impossible ou résultante d'un nouvel ordre des choses correspondant à un changement collectif des mentalités? 